\title{
Impact of a CMAM Intervention on Demand for Other Primary Health Care Services in Katsina State, Nigeria
}

\author{
Azeez Afebu Oseni ${ }^{1, ~ *}$, Wale Falade ${ }^{2}$ \\ ${ }^{1}$ Department of Public Health, University of Liverpool, Liverpool, United Kingdom \\ ${ }^{2}$ Reproductive Health Department, Obafemi Awolowo University, Ile Ife, Nigeria
}

Email address:

azeezoseni@gmail.com (A. A. Oseni), adewale.falad@gmail.com (W. Falade)

${ }^{*}$ Corresponding author

To cite this article:

Azeez Afebu Oseni, Wale Falade. Impact of a CMAM Intervention on Demand for Other Primary Health Care Services in Katsina State, Nigeria. American Journal of Biomedical and Life Sciences. Vol. 9, No. 5, 2021, pp. 248-253. doi: 10.11648/j.ajbls.20210905.14

Received: September 1, 2021; Accepted: October 9, 2021; Published: October 19, 2021

\begin{abstract}
Malnutrition in both acute and chronic forms is a global issue responsible for as much as $50 \%$ of infant mortality worldwide. Acute malnutrition exists as both moderate and severe forms depending on factors such as access to nutritious meals and maternal care practices. Moderate Acute Malnutrition (MAM) can be easily treated and has lesser mortality impacts compared to Severe Acute Malnutrition (SAM). Community Management of Acute Malnutrition (CMAM) is a communitybased approach to addressing SAM and MAM. In this study, the impact of a donor funded CMAM intervention delivered through primary healthcare (PHC) facilities in Katsina state, Nigeria is measured. Prior to this study, there was low utilization of these facilities due to the several factors such as transportation and communication problems, traditional conservatism, poor quality of services, and low literacy rates. A randomised sampling method was used to select and compare 5 CMAM and 5 non-CMAM facilities across the state. A test for significance for two independent groups (CMAM supported and non-CMAM supported sites) was also conducted for different categories of admissions such as antenatal care (ANC), outpatient department (OPD) and routine immunization (RI). From the result, it is concluded that the CMAM intervention increased the rate of patient access and use of PHCs within the state. The test of significance also shows significant differences between the admissions for OPD, ANC, and RI when compared to non-CMAM sites.
\end{abstract}

Keywords: Children Under 5 Years, Community Management of Acute Malnutrition, Antenatal Care, Outpatient Department, Routine Immunization, Severe Acute Malnutrition, Moderate Acute Malnutrition

\section{Introduction}

Community Management of Acute Malnutrition (CMAM) is the treatment of acute malnutrition using a communitybased approach that achieves wider access and coverage, and aims at early detection and referral of identified cases. Cases of Severe Acute Malnutrition (SAM) without medical complications or Moderate Acute Malnutrition (MAM) are treated with nutrient-dense foods and ready-to-use therapeutic foods to regain the lost nutrients due to malnutrition at the shortest possible time [1]. SAM cases have more negative impacts and affect an estimated 19 to 26 million children under 5 years globally and contribute to almost 1 million child deaths each year [2]. Other studies record that SAM kills children nine times more than nourished children, and MAM kills children three times more [3]. While CMAM has its origins in emergency contexts, non-emergency levels of SAM can be quite high, if not higher than in emergency settings $[2,4]$. As a result, CMAM is implemented through Primary Health Care (PHC) structure as an integrated process which can vary from context to context [5].

Malnutrition treatment programs are usually implemented in underdeveloped countries experiencing emergency famine by international organizations. This is to flatten the curve of the detrimental effects of hunger and starvation in the affected regions. Park et al argued that the same energy is not invested during non-emergency scenarios due to lack of skilled staff and infrastructure [6]. Luter et al also bemoaned the low coverage of interventions despite the daunting situations of hunger and starvation experienced by remote 
villages and dispersed communities of developing nations [7]. Hence, adequate aid support is not sent to the affected regions early enough, which invariably truncates the success of CMAM programs in such situations. Perry et al opined that early detection and engagement of target communities is key to an effective CMAM program [8]. According to Ubesie et al, there are widespread cases of malnutrition in Nigeria [9]. $60 \%$ of childhood deaths results from or are related to malnutrition while $25 \%$ are underweight [10]. These alarming statistics are not unconnected with the poor practice of childhood feeding in the infant years. Exclusive breastfeeding is rarely practiced $(15-17 \%)$, hence, subjecting the child to alternative feeding with less nutritional value and possible infection [11]. More than half of Nigerian infants are fed with complementary foods too early with resultant shortage in micronutrients such as iodine, iron, and vitamin A [12].

Records show that there is an increasing dearth in demand of PHC services in Nigeria, even though such services are epileptic and the PHCs are understaffed $[13,14]$. Reasons for low demand for services include: transportation and communication problems, traditional conservatism, poor quality of services, and low literacy rates [14]. Various strategies have been employed to address this gap including training and recruiting a large cadre of Community Health Workers (CHW), and the use of Village Health Committees (VHCs). However, it is proposed that large-scale donordriven interventions such as CMAM can have a significant impact on demand for other PHC services such as immunization, antenatal care (ANC), family planning, health promotion, and provision of essential drug supplies [15].

While PHC centres are relatively uniformly distributed throughout the Local Government Areas (LGAs) in Nigeria, the basic health services they provide are under-utilised [13]. Because of the large caseloads that CMAM generates, it clearly represents a unique opportunity to ramp up demand for other PHC services. This study will determine if the CMAM intervention in Katsina State (supported by Save the Children and funded by ECHO - the European Commission Humanitarian Aid Office) has a significant impact on demand for selected PHC services (routine immunisation, ANC and treatment of common ailments). A significant impact on demand would justify scaling up of CMAM, full integration of CMAM into health systems and its use as an entry point for PHC delivery as a whole within similar contexts.

\section{Research Method}

\subsection{Study Design}

Daura LGA in Katsina state was purposefully selected for the study as a major hub of CMAM intervention in the state. Based on the calculation from the sample size formula for comparing two independent groups, 5 facilities each were randomly selected from CMAM-implementing and non CMAM-implementing health facilities. Secondary data was sourced from NHMIS (National Health Management
Information System) monthly summary of PHC facilities. Data was collected from 4 sections of the monthly summary form namely: general attendance, ANC, immunization, and malaria testing (focus on under 5 children). Data on the 4 sections were collected from CMAM-implementing and non CMAM-implementing health facilities over a one-year period (January 2012 to December 2012).

\subsection{Sampling Method}

A stratified random sampling method was used. The first stage involved listing CMAM-implementing and non CMAM-implementing health facilities into 2 groups and using a simple random selection to choose 5 health facilities in each group. Overall, 10 health facilities were selected from CMAM-implementing and non CMAM-implementing health facilities. The sampling frame was the list of health facilities in Daura LGAs. The 10 facilities were drawn from the list of 22 health facilities in Daura LGA. Nine (9) of them was CMAM implementing sites while 13 was non-CMAM implementing sites.

\subsection{Sample Size}

This was determined using the formula for determining sample size for two equal groups with assumptions including power of small population $(Z)=90 \%$, corresponding to two tailed significance level $\left(Z_{\alpha / 2}, 1.96\right.$ for $\left.\alpha=.05\right)$, and ratio between study groups $=1$.

Sample size will be determined using the formula for determining sample size for two equal groups.

$$
\left.\mathrm{N}=\frac{(r+1)}{r} * \frac{\sigma^{2}\left(Z_{\text {power }}+Z \alpha / 2\right.}{d^{2}}\right)^{2}
$$

$\mathrm{N}=$ size for the group

$\mathrm{r}=$ ratio of larger group to smaller group

$\sigma=$ standard deviation (6.25)

$\mathrm{d}=$ clinically meaningful difference in means of outcome (2)

$\mathrm{Z}_{\text {power }}=$ corresponds to power $(90 \%)$

$\mathrm{Z}_{\alpha / 2}=$ corresponds to two tailed significance level (1.96 for $\alpha=.05$ )

Therefore, $\mathrm{N}=\frac{(1.56+1)}{1.56} * \frac{6.25^{2}(.9+1.96)^{2}}{2^{2}}$

$\mathrm{N}=6$ for each group

Sample size $(\mathrm{N})$ calculated from the formula was 6 for each group. However, 5 facilities were selected for each group because only 5 facilities in the non-CMAM implementing PHC sites had significantly complete monthly summary forms that were available and accessible. This was assumed not to significantly affect the power of the sample size.

Randomly selected sites

These are listed in the table below:

$\begin{array}{lll}\text { S/N } & \text { CMAM facilities } & \text { Non-CMAM facilities } \\ 1 & \text { PHC D/Kola } & \text { PHC Tambu } \\ 2 & \text { MCHC Kalgo } & \text { Kanti MCH } \\ 3 & \text { PHC Baraji } & \text { Madobi HC } \\ 4 & \text { PHC Hindatu } & \text { Urban HC } \\ 5 & \text { CHC Daura } & \text { PHC T/wada }\end{array}$




\subsection{Data Collection Process}

Secondary data was sourced from NHMIS monthly summary of PHC facilities. Data was collected from 4 sections of the monthly summary form namely, general attendance, ANC, immunization, and malaria testing (focus on under 5 children). The general attendance data included gender of patients and age groups as basic demographic characteristics. Data on the 4 sections was collected from CMAM-implementing and non CMAM-implementing health facilities over a one-year period (January 2012 to December 2012). Monthly summary forms for each of the 5 facilities were collected into Excel Sheet 2010. Data entry from source documents (registers) and cleaning will be done using SPSS ${ }^{\odot}$ (version 16).

\subsection{Analysis}

Analysis was done using SPSS $^{\odot}$ (version 16). The analysis involved using descriptive as well as bivariate level of analyses. The first stage involved using frequencies and tables to examine distributions and patterns while the student t-test $(95 \% \mathrm{CI})$ was used to test for significance for two independent groups (CMAM supported and non-CMAM supported sites).

\section{Results and Discussion}

Table 1. Demographic Characteristics of Children on Routine Immunization and Malaria Case Management.

\begin{tabular}{|c|c|c|c|}
\hline \multicolumn{2}{|c|}{ CMAM implementing facility } & \multicolumn{2}{|c|}{ Non-CMAM implementing facility } \\
\hline \multicolumn{2}{|c|}{ Outpatient attendance } & \multicolumn{2}{|c|}{ Outpatient attendance } \\
\hline Male & Female & Male & Female \\
\hline 7690 & 9620 & 2946 & 3233 \\
\hline \multicolumn{2}{|c|}{ Routine immunization (RI) } & \multicolumn{2}{|c|}{ Routine immunization (RI) } \\
\hline 6625 & 8353 & 4067 & 4281 \\
\hline \multicolumn{2}{|c|}{ Malaria testing } & \multicolumn{2}{|c|}{ Malaria testing } \\
\hline 4613 & 6594 & 5968 & 6123 \\
\hline
\end{tabular}

The total outpatient number accessing the facilities had
10,636 males and 12, 853 females. Non-CMAM sites had 2,946 males and 3,233 females for outpatient service. CMAM supported sites had 7,690 males and 9,620 females for outpatient service. The number of male children accessing routine immunization was 10,692 and 12, 634 females. CMAM supported sites had 6,625 males and 8,353 females. Non-CMAM sites had 4,067 males and 4,281 females. On malaria testing, 10,581 males and 12, 717 females had case management service on malaria. CMAM sites had 4,613 males and 6,594 females while non-CMAM sites had 5,968 males and 6,123 females respectively.

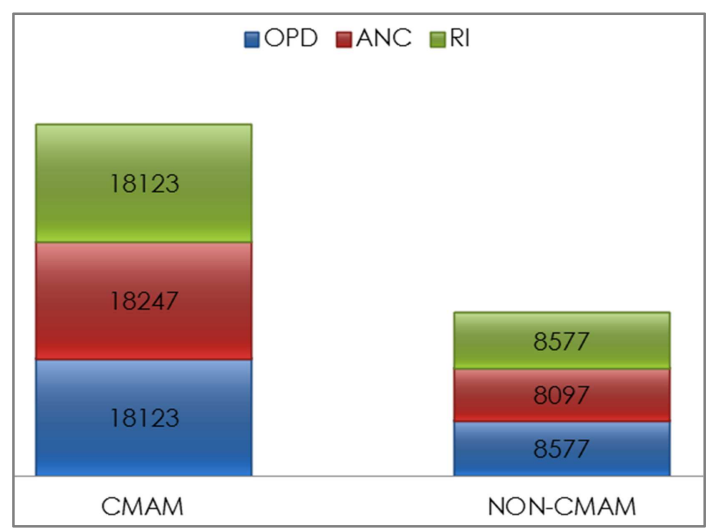

Figure 1. Number of patients accessing care in CMAM and Non-CMAM sites.

The Figure 1 above shows the number of patients for the outpatient department (OPD), women accessing antenatal care (ANC) and children attending the clinic for routine immunization (RI). The number of patients for OPD and those accessing ANC and RI for CMAM sites were higher compared to non-CMAM sites: OPD, ANC and RI for CMAM sites were $18,123,18,247,18,123$ respectively while OPD, ANC and RI for non-CMAM sites were 8,577, 8,097 and 8,577 respectively. The t-test of significance was compared in figures 2, 3 and 4 for each service (OPD, ANC and $\mathrm{RI}$ ) accessed at the CMAM and non-CMAM sites.

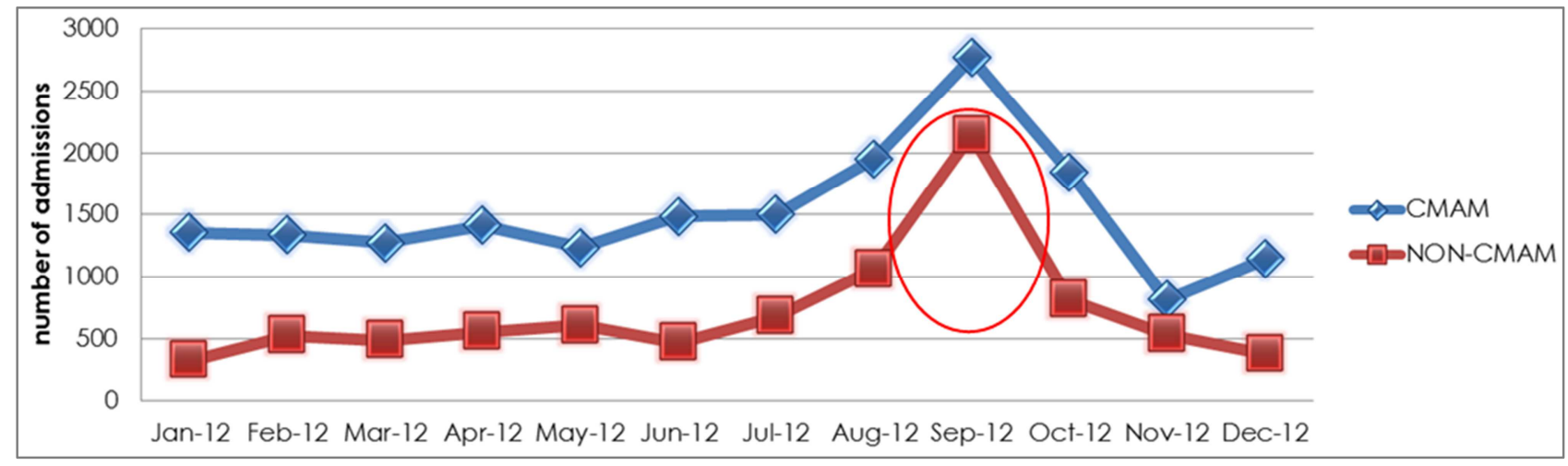

Figure 2. Admission trend for OPD in CMAM and Non-CMAM Sites from January 2012 to December 2012.

Figure 2 describes the trends in admissions from January to December 2012. The admission trend for CMAM and nonCMAM sites increased relatively from January up to September 2012 when admissions in both sites were its peak.
Admission trends declined up to November 2012 for both group of sites while that of non-CMAM sites continued declining and that of CMAM sites increased. The independent t-test results showed that there was a significant 
difference in admission trends when admission for OPD in $(\mathrm{t}=0.000575)$.

CMAM is compared to non-CMAM sites with $\mathrm{t}<=0.05$

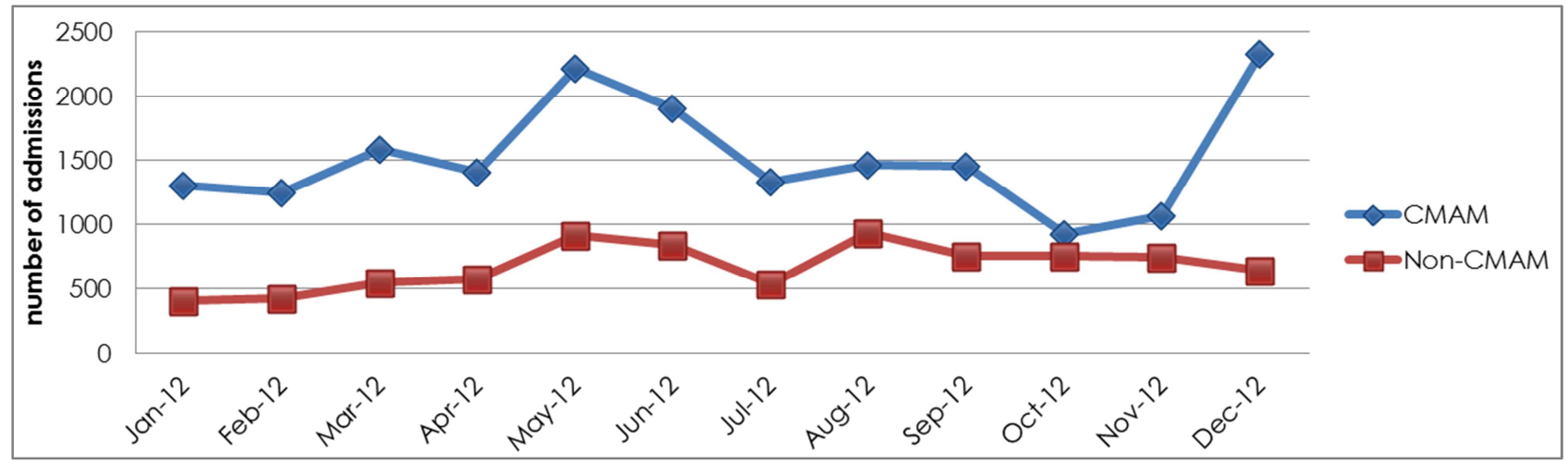

Figure 3. Trends in ANC from January to December 2012 in CMAM and Non-CMAM sites.

Figure 3 describes the trends in admissions for ANC service from January to December, 2012. The admission trends for both groups (CMAM vs non-CMAM) had similar variation pattern up to October 2012, with CMAM admissions relatively higher. However, the admission increased sharply for CMAM sites by November and
December, 2012 while the non-CMAM sites declined slightly. The independent t-test results showed that there was a significant difference in admission trends when admission for ANC in CMAM is compared to non-CMAM sites with $\mathrm{t}<=0.05(\mathrm{t}=0.001078)$.

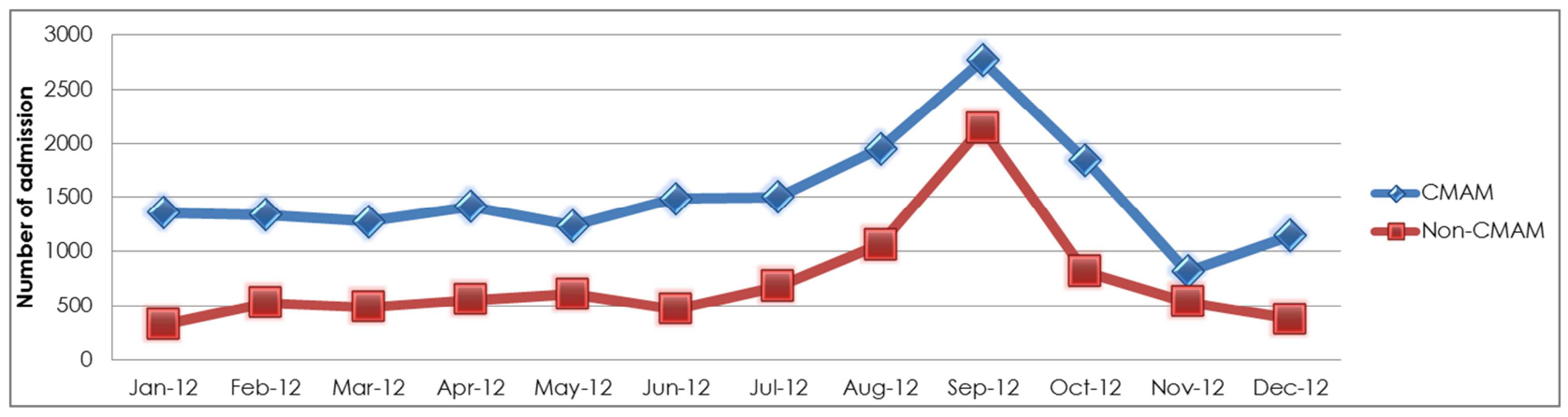

Figure 4. Admission trend for RI in CMAM and Non-CMAM Sites from January 2012 to December 2012.

Figure 4 describes the trends in admissions for routine immunization (RI) from January to December 2012. The admission trend for CMAM and non-CMAM sites increased relatively from January up to September 2012 when admissions in both sites were its peak. Admission trends declined up to November 2012 for both group of sites while that of non-CMAM sites continued declining and that of CMAM sites increased. The independent t-test results showed that there was a significant difference in admission trends when admission for OPD in CMAM is compared to non-CMAM sites with $\mathrm{t}<=0.05(\mathrm{t}=0.000575)$.

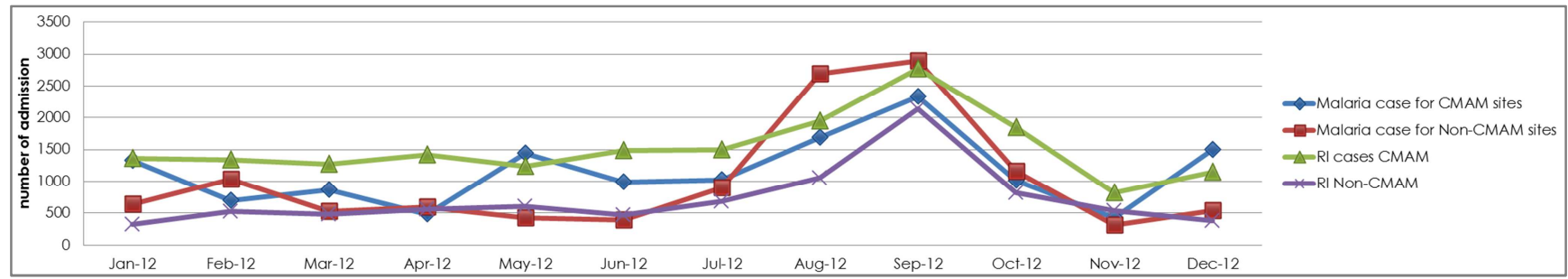

Figure 5. Admission trends for RI and Malaria in CMAM and Non-CMAM sites.

Figure 5 shows admission trends for RI and malaria cases for CMAM and non-CMAM sites. The trends appear to be congruent both in CMAM and non-CMAM sites. Malaria surged highest in the month of September, 2012 which is in keeping with the expectation that malaria cases are highest during the peak of the rainy season. Also, the number of admissions for malaria was higher in CMAM sites compared to non-CMAM sites. However, there was no significant difference $\mathrm{t}>=0.05(\mathrm{t}=0.653532)$. Because interventions such as CMAM improve the quality of care of PHCs by providing 
supply chain support, equipment and materials, staff capacity building and monitoring and data systems support, the PHCs become more attractive to patients. This can improve the status of PHCs and increase patient attendance as discussed by Olalubi and Bello [16].

Integrating vertical donor-funded programs into existing primary health systems can be both challenging and rewarding and despite some critics warning that such integration can weaken health systems if not properly managed, this study finds that there is enough justification for integration [17]. Because nutrition is probably the most important component of primary health care, donor-funded nutrition programs therefore represent a promising opportunity to boost coverage and reach of PHC programs through improving service delivery quality [18].

\section{Conclusion and Recommendations}

The trends of admission for CMAM and non-CMAM sites have been observed across selected primary healthcare facilitates in Katsina state. This was done to ascertain the impact of a community nutrition (CMAM) intervention across the state. From the results, it is concluded that the CMAM intervention has at least doubled the rate of patients' access of PHC facilities within the state. The t-test also shows higher peaks in CMAM facilities and significant differences between the admissions for OPD, ANC, and RI when compared to non-CMAM sites. Public health interventions such as CMAM represent an excellent opportunity to boost demand for other primary healthcare services. There is clear evidence that donor-funded nutrition projects can have a beneficial effect on PHC attendance and coverage. Specific recommendations include:

1) Future nutrition programs funded by donors should have an explicit objective of improving PHC service delivery in related components including $\mathrm{ANC}$ and routine immunization

2) Further research should be conducted on the specific domains of health systems strengthening and the impact that integration with donor-funded projects can have

3) CMAM interventions should be intentionally designed to boost ANC and routine immunization outcomes

4) Governments should work closely with donors and design interventions targeting $\mathrm{PHC}$ to ensure approaches are synergized and outcomes are optimized.

5) Where CMAM programs exist, they should be scaled up to cover more primary health care centers bearing in mind they additional benefits that they provide.

\section{References}

[1] Mustafa, Y. K., Muazu, Y. U., Bolori, M. T., Muhammad, A. I., Aminu, B., \& Isa, Y. (2017). Community Management of Acute Malnutrition in Katsina State, Nigeria: A Report From 2010 To 2013. Mediterranean Journal of Basic and Applied Sciences (MJBAS), 1 (1), 155-163.
[2] UNICEF, (2012). UNICEF World Bank Joint Child Malnutrition Levels and Trends in Child malnutrition available www.who.int/nutgrowthdb/jms_unicef_who_wbpdf.

[3] Prendergast, A. J. (2015). Malnutrition and vaccination in developing countries. Philosophical Transactions of the Royal Society B: Biological Sciences, 370 (1671), 20140141.

[4] Deconinck, H., Swindale, A., Grant, F., \& Navarro-Colorado, C. (2008). Review of community-based management of acute malnutrition in the postemergency context: synthesis of lessons on integration of CMAM into national health systems in Ethiopia, Malawi and Niger. Washington: FANTA AED.

[5] Kautzky, K., \& Tollman, S. M. (2008). A perspective on primary health care in South Africa: Primary health care: In context. South African health review, 2008 (1), 17-30.

[6] Park, S. E., Kim, S., Ouma, C., Loha, M., Wierzba, T. F., \& Beck, N. S. (2012). Community management of acute malnutrition in the developing world. Pediatric gastroenterology, hepatology \& nutrition, 15 (4), 210.

[7] Lutter, C. K., Daelmans, B. M., de Onis, M., Kothari, M. T., Ruel, M. T., Arimond, M.,... \& Borghi, E. (2011). Undernutrition, poor feeding practices, and low coverage of key nutrition interventions. Pediatrics, 128 (6), e1418-e1427.

[8] Perry, H. B., Zulliger, R., \& Rogers, M. M. (2014). Community health workers in low-, middle-, and high-income countries: an overview of their history, recent evolution, and current effectiveness. Annual review of public health, 35, 399-421.

[9] Ubesie, A. C., \& Ibeziakor, N. S. (2012). High burden of protein-energy malnutrition in Nigeria: Beyond the health care setting. Annals of medical and health sciences research, 2 (1), 66-69.

[10] Babatunde, R. O., Olagunju, F. I., Fakayode, S. B., \& SolaOjo, F. E. (2011). Prevalence and determinants of malnutrition among under-five children of farming households in Kwara State, Nigeria. Journal of Agricultural Science, 3 (3), 173-181.

[11] Odunayo, S. I., \& Oyewole, A. O. (2006). Risk factors for malnutrition among rural Nigerian children. Asia Pacific Journal of Clinical Nutrition, 15 (4).

[12] Abeshu, M. A., Lelisa, A., \& Geleta, B. (2016). Complementary feeding: review of recommendations, feeding practices, and adequacy of homemade complementary food preparations in developing countries-lessons from Ethiopia. Frontiers in nutrition, 3, 41.

[13] Abdulraheem, B. I., Oladipo, A. R., \& Amodu, M. O. (2012). Primary Health Care Services in Nigeria: Critical Issues and strategies for enhancing the use by the rural communities.

[14] Oyekale, A. S. (2017). Assessment of primary health care facilities' service readiness in Nigeria. BMC health services research, 17 (1), 1-12.

[15] Adeyemi, O., Afolabi, W. A., Ferguson, E., Akinyele, I., Lawal, H., Okunola, R. A., \& Yohanna-Dzingina, C. (2014). How to strengthen the CMAM programme in Northern Nigeria and reduce rates of programme defaulting.

[16] Oluwasogo A. OLALUBI, Sebutu I. BELLO (2020). Community-Based Strategies to Improve Primary Health Care (PHC) Services in Developing Countries. Case study of Nigeria. 
[17] Chaitkin, Michael, Nathan Blanchet, Yanfang Su, Rebecca Husband, Pierre Moon, Andrea Rowan, Steve Gesuale, Candice Hwang, Paul Wilson, and Kim Longfield. (2019) Integrating Vertical Programs into Primary Health Care: A Decision-Making Approach for Policymakers. Washington, DC: Results for Development.
[18] Christian Kraef, Benjamin Wood, Peter von Philipsborn, Sudhvir Singh, Stefan Swartling Peterson \& Per Kallestrup (2020) Primary Health Care and Nutrition. 